\section{Triglyzerid-Transferprotein, mikrosomales}

\author{
K. J. Lackner ${ }^{1}$ und D. Peetz ${ }^{2}$ \\ ${ }^{1}$ Institut für Klinische Chemie und Laboratoriumsmedizin, \\ Universitätsmedizin Mainz, Mainz, Deutschland \\ ${ }^{2}$ Institut für Labormedizin, Helios Klinikum Berlin-Buch, \\ Berlin, Deutschland
}

Synonym(e) Mikrosomales Triglyzerid-Transferprotein; MTP

Englischer Begriff microsomal triglyceride transfer protein

Definition Transferprotein, das neutrale Lipide zwischen Membranen und Lipoproteinpartikeln, vor allem neu synthetisierte VLDL ( $\triangleright$ Very low density lipoprotein) (Leber) bzw. $\checkmark$ Chylomikronen (Darm), transferiert.

Beschreibung MTP ist ein mikrosomal lokalisiertes heterodimeres Protein, das neutrale Lipide transportiert. Es besteht aus einer großen 97-kDa-Untereinheit, die für die Aktivität entscheidend ist und einer 55-kDa-Einheit, die identisch mit der Proteindisulfidisomerase ist. Die Lipidtransferaktivität ist unverzichtbar für die normale Synthese triglyzeridreicher - Lipoproteine. Genetische Defekte der 97-kDa-Untereinheit führen zur A- $\beta$-Lipoproteinämie, einer autosomal rezessiven Erkrankung, bei der die ApoB-haltigen $\triangleright$ Lipoproteine fehlen mit der Folge eines gestörten Transportes von Neutralfetten und fettlöslichen Vitaminen. Der MTP-Inhibitor Lomitapid ist in einigen Ländern zur Therapie der homozygoten familiären Hypercholesterinämie trotz signifikanter Nebenwirkungen (u. a. Transaminasenanstieg) zugelassen.

\section{Literatur}

Barter PJ, Rye KA (2016) New era of lipid-lowering drugs. Pharmacol Rev 68:458-475

Hussain MM, Shi J, Dreizen P (2003) Microsomal triglyceride transfer protein and its role in apoB-lipoprotein assembly. J Lipid Res $44: 22-32$ 